எதிர் வழக்காறுகள்

தி. உமாதேவி அ,.

அ நவீன இந்திய மொழிகள் மற்றும் இலக்கியத் துறை, தில்லி பல்கலைக்கழகம், புதுடில்லி-110021, இந்தியா

\title{
Counter-Narrative Tradition
}

\section{Umadevi a,*}

a Department of Modern Indian Languages and Literary Studies, University of Delhi, New Delhi-110021, India.

* Corresponding Author: dumadevi@mil.du.ac.in

Received: 30-04-2021 Revised: 22-07-2021

Accepted: 23-07-2021

Published: 26-07-2021

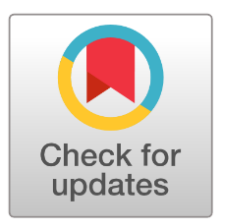

\begin{abstract}
The term "counter narrative" refers to a narrative that takes on meaning through its relation with one or more other narratives. While this relation is not necessarily oppositional, it involves a stance toward some other narrative(s), and it is this aspect of stance, or position, that distinguishes counter narrative from other forms of intertextuality. The article explained, "counter-narratives only make sense in relation to something else, that which they are countering counter narratives has been seen as a means of opposing or resisting socially and culturally informed master narratives (about, for example, skin colour, ethnicity, and food culture), which are often normative or oppressive, or exclude perspectives or experiences that diverge from those conveyed through master narratives. In this sense, counter narratives play a role in storytellers positioning themselves against, or critiquing, the themes and ideologies of master narratives. Used in this way, "counter narratives" refer to "the stories which people tell and live which offer resistance, either implicitly or explicitly, to dominant cultural narratives" This articles explains the counter narratives on perception of black skin colour and food culture. Both the concepts of counter-culture and counter-narrative tradition are new in the folklore field of Tamil traction
\end{abstract}

Keywords: Counter Narrative, Intertextuality, Perception of Black Colour, Counter Story, Counter-Narrative Tradition

முன்னுரை

எதிர்க்கருத்தாடல் என்பது ஒரு தத்துவக் கருத்தோ அல்லது வேறெந்தக் கருத்தோ அல்ல, அது ஒரு கதையாடல் வகைமை என்பதைக் கருத்தில் கொண்டால், இது ஒரு நாட்டுப்புற கதையாடல் வகைமையாக எவ்வாறு பரிணமிக்கிறது என்பது விளங்கும். இவ்வாறான எதிர்க்கருத்தாடல்களை உருவாக்குவோர் சமூகப் பண்பாட்டைக் கட்டமைப்பவர்களாகவும் அந்த நிலப்பரப்பில் தத்தம் வழிகளைக் கண்டடையும் யத்தனங்களோடும் இயங்குகிறார்கள் என்பது தெளிவு .9வர்கள் தமது அடையாளங்கள் பற்றியப் பார்வைகளின் விழுமியங்களை உருவாக்கிக் கொள்கிறார்கள் (Stephen, 1999). திருநெல்வேலி மாவட்டத்தில் சேகரிக்கப்பட்ட கள ஆய்வுத்தரவுகளின் அடிப்படையில் இக்கட்டுரை அமைகிறது. 


\section{நிறம் சார்ந்த சிந்தனைகள்}

ஒவ்வோர் இனத்திற்கும் அதன் நிலம்சார்ந்த உணவு அடையாளங்கள் உண்டு. மனித சமூகங்கள் தங்கள் வாழ்விடம், சுற்றுச்சூழல், இயற்கை வளம் ஆகியவற்றின் அடிப்படையில் தமது உணவுப் பழக்கத்தை ஏற்படுத்திக் கொண்டுள்ளன. உருவ அமைப்பும் நிறமும், உண்ணும் விதமும் என ஒவ்வொரு சமூகக் குழுவிற்கும் பல தனித்தன்மைகள் உள்ளன. இத்தனித்தன்மைகளைச் பாதுகாக்கும் பல கதையாடல்கள் வாய்மொழியாக வழக்கில் உள்ளன. இவர்களின் சமூக அமைப்மையும், இவர்களுக்குள் உள்ள ஏற்றத்தாழ்வையும் முறியடிக்கும் வகையில் அவற்றைப் புனைகின்றனர். இது தமிழ்ச் சமூகத்திற்கும் பொருந்தும். மனிதன் உருவம் நிறம் மீதான விலக்கும்-விருப்பமும் பல வகையில் வலுப்படுத்தியுள்ளதற்குச் சூழலியல் காரணிகள் அடிப்படையாய் உள்ளன. இதன் வாயிலாக நமது இனத்தின் சிறப்புத்தன்மை, வரலாறு, அதன் சமூக அரசியல் அடையாளம் ஆகியவற்றைக் குறித்த அறிவியல் அடிப்படையிலான விவாதத்தை வளர்த்தெடுக்க வேண்டிய அவசியம் உள்ளது. அப்பணியைப் பண்பாட்டு ஆய்வுகளின் வழி முன்னெடுக்க முடியும். பண்பாடுகள் பன்முகத் தன்மையானவை. கறி, மீன் சாப்பிடும் இயற்கை உணவு விரும்பிகளுக்கும், குறிப்பிட்ட உணவுப் பழக்கம் கொண்ட இலை-தழை, காய்கறிகளை மட்டுமே உண்பவர்களுக்கும் பொதுவானதாகத் தமிழர் உணவு உள்ளது. நம் மீது ஆதிக்கம் செலுத்தும் பண்பாட்டின் பொருண்மைகள் எவை என்பவை குறித்தும் அவற்றை எதிர் கொள்வதற்கானப் பல கருத்துக்களை வெளிப்படுத்தும் இக்கதையாடல்கள் கீழே விளக்கப்படுகின்றன. கருப்பு, சிவப்பு, பச்சை, பொன் நிறம் என்பது தமிழ் மக்கள் வழக்கில் நெடுங்காலமாக அறிந்திருந்த நிறங்கள் (Paramasivan, 2009). மஞ்சள் என்பதை பொன்நிறம் என்று குறிப்பிட்டார்கள். சந்தனம், தாழம்பூ எல்லாம் பொன் நிறத்தில்அடக்கம் வெங்காய நிறம், பாசிப்பச்சை நிறம், மரக்கலர், சாணிப் பச்சைநிறம், வெந்தயக்கலர், பாசிப் பச்சைக் கலர் என பல வண்ணங்கள் வழக்கில் உண்டு. வெள்ளையும் தமிழ் மக்கள் அறிந்திருந்த நிறந்தான். அது உண்மை, தாய்மை, எளிமை ஆகியவற்றின் குறியீடாகப் பயன்பட்டது. கருப்பு என்றோ சிவப்பு என்றோ சொல்ல முடியாத நிறம் மா நிறமாகியது. தமிழ் மக்களின் அடிப்படை நிறம் கருப்பு. அது அவர்களின் பொது நிறம். அதாவது தோலின் நிறம்.

\section{இலக்கியங்களில் கருப்பு நிறம்}

கருப்பு என்பது தொல்காப்பியத்தில் நிறம் என்ற பொருளிலேயே இடம் பெற்று உள்ளது இரண்டு நிறங்களைப் பற்றியே தொல்காப்பியர் பேசுகிறார் கறுப்பும் சிவப்பும் வெகுளிப் பொருள நிறத்துரு உணர்த்தற்கும் உரிய என்ப கறுப்பு, சிவப்பு என்பன சினத்தை உனாத்தும் சொற்களாகவும் வரும் என்பது தொல்காப்பிய இலக்கணமாகும். 'திருக்குறளில்' கறுத்து என்ற சொல் சினந்து என்ற பொருளைத் தருகிறது பக்தி இலக்கியங்களில் கறுப்பு அழகுக்குரிய நிறம். அது ஒளி வீசும் என்பது திருமாலை ஆண்டாள் கண்ணன் என்னும் கருந்தெய்வம் காட்சி பழகிக் கிடப்பேன் என்கிறார். ஆழ்வார்கள் பலரும் திருமாலைக் கரிய மாணிக்கம் என்று பாடியுள்ளனர். இராமனது கரிய உடம்பிலிருந்து ஒளி கிளர்ந்தது என்ற செய்தியை 'வெய்யோன ஒளி தன் மேனியின் விரிசோதியின் மறைய' என்று கம்பர் பாடுகிறார் கண்ணப்பர் பிறந்தபோது அவரது கறுத்தமேனி ஒளியுடையதாக இருந்தது என்பதனைக் 'கருங்கதிர் விரிக்கும் மேனி காமருகுழவி என்று பாடுகிறார் சேக்கிழார் தன்மீது பட்ட ஒளியைப் பளபளப்புடைய கருப்புநிற மனிதத்தோல் எதிரொளி செய்து காட்டும் என்று கம்பரும் சேக்கிழாரும் பாடுகின்றனர். நன்னூல் கண் என்னும் வேற்றுமை உருபினை விளக்குவதற்கு கறுப்பின் மிச்குள்ளது அழகு என்பதாகும். கறுப்பு அழகோடு சேர்த்து எண்ணப்பட்ட கறுப்பு நிறம் பின்னர் தனது மதிப்பை இழந்து அழகின்மை என்பதற்கு அது மாறிப் போனது. இதற்கான காரணத்தை அதிகாரம் சார்ந்த அரசியல் அமைப்புக்குள்ளே தேட வேண்டும். ஐரோப்பியப் பாதிரிமார்கள், பிராமணர்கள் ஆகிய அனை வரும் தமிழர்களை விடச் சிவந்த நிறம் உடையவர்கள். அவர்கள் ஐந்து நூற்றாண்டுக்கு மேலாகத் தமிழ்நாட்டில் அரசியல் அதிகாரமும், அரசியல் சித்தாந்தங்களையும் நடைமுறைகளையும், உயர்த்திப் பிடித்தவர்கள். ஆன்மீக அதிகாரமும் சிவந்த நிறமுடையவர்களின் கையிலேயே இருந்தது. எனவே இந்த நிறம் அதிகாரத்தின் நிறமாக, உயர்ந்த ஆன்மீகத்தின் நிறமாக, மேட்டிமையின் சின்னமாக, அழகு 
நிறைந்ததாகக் காட்டப்பட்டது. சுருக்கமாகச் சொல்வதானால், தமிழ் பேசும் பெருவாரியான மக்கள் கூட்டத்தாரின் மரபுவழி அழகுணர்ச்சி மனிதத் தோலின் நிறத்தைப் டொறுத்தமட்டில் திசைமாற்றம் பெற்து. நிலையில் சொல்வதானால், கறுப்பு நிறமுடைய மக்கள் அழகற்றவர்களாகவும், ஆளப்படுபவர்களாகவும் அதிகாரத்திற்குத் தகுதியற்றவர்களாகவும் காட்டப்பட்டனர். ஆனால் பண்பாட்டு வளர்ச்சியில், கருப்பு ஒதுக்கப்பட்டு விட்டது. கருப்பு நிறம், தாழ்வு, இழிவு, துக்கம் என்பதின் அடையாளமாக மாறிவிட்டது. இதை மற்றவர்கள் சொல்லிச் சொல்லி கருப்பர்களே அவ்வாறு உணர ஆரம்பித்துவிட்டனர். கருத்தைச் சொல்லுவதற்கு தோலின் நிறம் குறியீடாகப் பயன்படுத்தப்படுகிறது. இன்று கறுப்பு, சிவப்பு ஆகிய இரண்டு நிறங்கள் கீழ்ச்சாதிக்காரன். மேல்சாதிக்காரன் என்பதைக் குறியீடுகளாகச் சுட்டி நிற்கின்றன. சமூக முரண்பாடுகள் மனிதனின் தோலின் நிறத்தைக் கொண்டு வெளிப்படுகின்ற வழக்கம் உருவாகியது. எனவே கருப்பின் இடத்தை நீலம் பிடித்துக் கொண்டு மேலே வந்துவிட்டது. அதாவது ருப்பின் வளர்ச்சி நீலம்.

\section{இதிகாசங்களில் கருமை}

இந்தியப் புாணங்களிலும் கதைகளிலும் கருப்பு நிறப் பெண்கள், அவர்களின் நிறத்துக்காகவே கொண்டாடப்பட்டிருக்கிறார்கள். மகாபாரதத்தில் பாண்டவர்களின் மனைவியான திரௌபதி, கருப்பு நிறத்திலான தனது தோல் மீது பெருமை கொண்டவளாக இருக்கிறாள். காவியத்தில் திரௌபதி, யாக அக்னியில் பிறந்தவள் என்பதால் யாகசேனி என்றும் கரிய நிறத்தவர் என்பதால் கிருஷ்ணை என்றும் பாஞ்சால நாட்டு இளவரசி என்பதால் பாஞ்சாலி என்றும் அழைக்கப்பட்டார். திரௌபதி, பாஞ்சால நாட்டு அரசர் துருபதன் செய்த யாகத்தில் தோன்றியவர். இவருடன் திருத்துமன் என்னும் சகோதரனும் யாகத்தீயில் தோன்றினார். திரௌபதி கரிய நிறத்தவர், அழகில் சிறந்தவள். தெய்வங்களைப் பேசும் இடத்தில் அவற்றின் நிறங்கள் பேசப்படுகின்றன. மாயோன் மலை போன்று நீலநிறத்தில் இருக்கிறான் பலராமன் பலராமன் (வாலியோன்) அருவிபோல வெள்ளை நிறத்தில் இருக்கிறான் என்று ஒரு சங்கப் பாடல் கூறுகிறது. திருமாலுக்கும் பலராமனுக்கும் நிறம் சொல்லப்பட்டாலும், முருகன், கொற்றவை போன்ற தெய்வங்களுக்கு நிறம் சொல்லப் படவில்லை. சிவபெருமாளின் கழுத்து நஞ்சுண்ட காரணத்தால் கருமையும் நீலமும் கலந்த வண்ணத்தில் அமைந்திருப்பதாக மற்றொரு பாட்டு கூறும் பக்தி இலக்கியக் காலந்தொட்டு தெய்வங்களுக்கும், மளிதர்களுக்கும் பல்வேறு நிறங்கள் போப்படுகின்றன வுமன் ஆஃப் வொர்த் (Women of Worth) என்ற அமைப்பின் நிறுவனர் கவிதா இமானுவேல், நிறப்பாகுபாட்டுக்கு எதிராக ஒரு இயக்கத்தை முன்னெடுத்தார் Dark is Beautiful 'Stay Unfair, Stay Beautiful' என்ற கோஷத்தை வெளியிட்டார்.

\section{எதிர் நாட்டார் வழக்காறுகள்}

எதிர் அழகியல் என்பது ஏற்கனவே உருவாக்கப்பட்டுள்ள சிந்தனையில் இருந்த மாறுபட்ட ஒரு கருத்தை விளக்குவது கருப்பு நிறம் கொண்ட உடல் அழகற்றது என்ற பொருண்மை கொண்டக் கருத்தாடல்கள் மக்களிடம் வழக்கில் இருக்கின்றன. அவர்கள் கருப்பாய் இருக்கும் பெண்களைக் "காக்கா கருப்பு" அண்டங்காக்கா கருப்பு நனைந்த பனைக்கருப்பு, என்றெல்லாம் கூறுவர். திருமணத்திற்கு பெண் பார்த்து விட்டு வந்த இரு பெண்களின் உரையாடல் இது. பொண்ணு பார்ப்பதற்கு எப்படி இருப்பாள் என்று கேட்டதற்கு இருப்பாள் இருப்பாள் இருட்டுக்கு பொட்டு வைத்த மாதிரி இருப்பாள் என்றும் கேலியாகப் பதில் சொல்வது கிராம மக்களின் வழக்கமாக இருந்து வருகிறது. அதே நேரத்தில் அந்தக் கேள்விக்குப் பதிலாகக் கருப்பு நிறம் அழகற்றது அசிங்கமானது என்ற கதையாடலை மறுத்து ஒரு புனைவின் வழி தங்களது எதிர்ப்பைப் பதிவு செய்வதையும் காணமுடியும். இது எதிர் அழகியல், எதிர் கலாச்சாரம் என்று சொல்லப்படுகிறது. அதன் அடிப்படையில் இந்த கதையாடல் சான்றாக, காட்டப்படுகிறது. ஒரு பெண் கருப்பு என்று அவமானப்படுத்தப்பட அதற்காக எதிரான ஒரு கருத்தாடலை உருவாக்குகிறார் தகவலாளி. அந்த கருத்தாடலில் என்னுடைய கருப்பு உன் உச்சந்தலையில் இருக்கு உனது சிவப்பு என் உள்ளங்காலில் இருக்கு என்று பதிலடி கொடுக்கின்றார். இதுபோன்று பல மரபுத்தொடர்கள் வழக்கில் உள்ளன. காந்தலே ருசி, கருப்பே அழகு என்பது போன்ற பழமொழிகள் உண்டு கருப்பான பெண்ணிற்கு நகை போட்டு அழகு பாரு என்றும் 
சிவப்பான பெண்ணிற்கு நகைகளை போட்டுச் செருப்பால் அடி என்று பல்வேறு பழமொழிகளும் உண்டு அவ்வாறெனில் கருப்பாய் இருப்பவர்கள் அழகற்றவர்கள் அல்லர் என்று கூற எதிர்வழக்காற்று மரபு பயன்படுத்தப்படுகிறதா? எதிர்க்கதை மரபை விளக்குவதன் மூலம் இக்கேள்விக்கு விடையளிக்க இயலும்.

மேலும் சிவப்பான பெண்ணிற்கும் நிழல் கருப்பாகத்தான் இரருக்கும். இதற்கு வலுச் சேர்க்கும் விதமாக ஒரு கதையும் இங்கு விளக்கப்படுகிறது. இறைவன் உலகத்தை படைக்க விரும்புகிறார் இறைவன் அவ்வாறு படைக்கும் போது மனிதர்களை கோதுமை மாவினால் படைக்கிறார் அதாவது கோதுமை மாவை பிசைந்து ரொட்டி செய்கிறார். அதில் சரியாக வேகாமல் சில சப்பாத்திகளைச் செய்து விடுகிறார். அந்த சப்பாத்திகள் மனிதர்களாக மாறுகின்றன. சரியாக வேகாத சப்பாத்திகள் வெண்மை நிறம் கொண்ட மக்களாக உருவானார்கள் அவர்களே மேற்கத்தியர்கள், வடஇந்தியர்கள் சில சப்பாத்திகளை சுடும்போது அதிகமாக வேக வைத்து விடுகிறார் .அவை கரிந்துப் போய் விடுகின்றன. அவை ஆப்பிரிக்க இன மக்களாகக் மாறி விட்டனர் என்றும் சரியான பக்குவத்தோடு வேக வைத்து உருவாக்கப்பட்ட சப்பாத்தி மாவு ரொட்டிகள் மாநிறம் கொண்ட மக்கள் இந்தியர்கள், தமிழர்கள், திராவிடர்கள் என்ற கதை வழக்கில் இருந்து வருகிறது. கருப்பு பெண்களை தமிழகத்தில் உள்ள கிராமங்களில் பெண் மாநிறம், புதுநிறம் என்றெல்லாம் குறிப்பிடுவார். மாமை என்பது மருவி மாநிறம் என்றாகிவிட்டது. இதை வட இந்தியாவில் சாவ்லா நிறம் என்பர் இந்த கதையாடல்கள் எவ்வாறு தங்களது கருத்தை தங்களின் எதிர்ப்பை உறுதிப்படுத்திக் கொள்வதற்கு மிகச் சிறப்பாக வழிவகுக்கின்றன மேலும் ஒருநாட்டுப்புறக் கதையின் வழி கருப்பு நிறம் கருப்பு நிறத்தின் சிறப்பை விளக்கும் முகமாகச் சான்றாகக் கூறப்படுகிறது இரு பெண்கள் சிறந்த நண்பர்கள் ஒன்றாய் வாழ்ந்து வந்தனர். ஒரு பெண் பார்ப்பதற்கு சிவந்த நிறம் கொண்டவள் இன்னொரு பெண் கருப்பு நிறம் கொண்டவள் இந்த இரண்டு பெண்களில் சிவந்த நிறம் கொண்டவள் எப்பொழுதுமே தன்னுடைய கலரின் மீதும் அழகின் மீது கர்வம் கொண்டு கருப்பாக இருக்கும் பெண்ணை கேலி செய்வாள் நீ கருப்பாக இருக்கிறாய் என்றும் அதனால் நீ அழகாக தெரியவில்லை என்றும் அடிக்கடி குறிப்பிடுவாள். அதற்கு எந்தப் பதிலையும் சொல்லாமல் மிகவும் பொறுமையுடன் தன்னுடைய காலத்தை கடத்திக் கொண்டு வந்தாள். ஒரு நாள் தன் தோழியின் கேலியைப் பொறுக்க முடியாமல் சரியான தனது பதிலின் வாயிலாக வாயை மூட வைத்தாள். அவளின் பதில் இதுவே. உன் முகத்தில் என் கருப்பு நிறம் மச்சமாக இருக்கும் பட்சத்தில் உனது சிவந்த முகம் மேலும் அழகாகக் காட்சியளிக்கும். நீ அழகுபடுத்தபடுவாய் ஆனால் அதே உனது சிவப்பு நிறத்தின் ஒரு புள்ளி எனது கருப்பு முகத்தில் ஏற்பட்டால் அது வெண்குஷ்டம் என்று நோயாக பார்க்கப்படும் எனவே இந்தக் கருப்பு எனது கருப்பு உனக்கு அழகை கொடுக்கும் உனது வெண்மை எனக்கு அருவருப்பை கொடுக்கும் என்று தன் பக்கத்து நியாயத்தை உறுதியாகக் குறிப்பிட்டாள். இங்கு அந்தப் பெண் எடுத்துரைத்த அனுபவக்கதை மரபுவழியான நம்பிக்கைக்கு எதிரானது என்பதில் ஐயமில்லை. இவ்வாறே மரபு வழியான நம்பிக்கைகளுக்கும் பழக்க வழக்கங்களுக்கும் சமூக வளர்ச்சிப் போக்கில் பல எதிர்வழக்காறுகள் உருவாகிய வண்ணமிருக்கின்றன எதிர்ப்புக்குரல்கள்தான் நாட்டுப்புறச் சிந்தனைப் பண்பாட்டின் இன்றியமையாத அம்ம் என்பத இந்தக்கதையாடல்கள் உறுதிப்படுத்துகின்றன.

ஒவ்வொரு சமூகக் குழுவிற்கும் பல தனித்தன்மைகள் உள்ளன. இத்தனித்தன்மைகளைச் பாதுகாக்கும் பல கதையாடல்கள் உண்டு இவர்களின் சமூக அமைப்பையும், இவர்களுக்குள் உள்ள ஏற்றத்தாழ்வையும் முறியடிக்கும் வகையில் புரிந்து கொள்ள முடியும். இந்த அடிப்படையில் புனையப் படும் கதையாடல்கள் சமூகத்தினரால் எளிதில் ஏற்றுக் கொள்ளப்படும் முதலாவதாக, மொழியைப் பயன்படுத்தலில் உள்ள செயல்ப்பட்டு முறையில் புராதன குல, குறி சின்னங்களுக்கு நிகரானதாக பேச்சு வழக்கேயாயினும் சமூக பொதுவுக்கு கொண்டு வந்து வார்த்தைகளின் பிரயோகங்கள் வழி அதிர்ச்சியளித்து கொண்டு அதை மரபாக மாற்றிவிட வேண்டும். அப்படியாகும் போது சொல்லுக்கும் பொருளுக்குமான இடுகுறிதன்மை புதிய சொல்லாடல்களுக்கு வழிகோலுவதாக அமைந்து மொழியில் புது மாற்றத்தை உருவாக்கும். அப்போது சமூக பொதுபுத்தியில் ஏற்கனவே நிலைகொண்டுள்ள மொழி அமைப்புகளில் புதிய பரிமாணத்தை அளிக்க முடியும். இரண்டாவதாக உண்மை, அழகு, இன்பம் என்ற மரபார்ந்த கலை இலக்கியத் திறன் என்ற ரசனையின் பன்முகத்தன்மைப் பற்றிய புதியப் புரிதலில் அதிகப் பொருண்மைகளுக்கு ஆக்கபூர்வமான முறையில் திறனாய்வை வரையறை செய்ய முயலும். மேட்டிமைதனம் கொண்ட உண்மை, அழகு, இன்பம் புற உலகில் எண்ணற்ற வழிகளில் இயங்கிக் கொண்டிருக்கும் ஆளுமைப் பொருண்மைகமை நின்று நிதானித்து அணுகும் தன்மை உருவாகிட மாற்று முறைகளிலான ஆக்கங்கள், பார்வைகள் 
முக்கியமானவையாகும். பெரும்பான்மையானவர்களின் அற விழுமியங்களுக்கு மாற்றாக அவர்களின் நடைமுறை உண்மைகளுக்கு எதிரான தொனியில் பயணப்படும் ரசனையை வளர்தெடுக்க வேண்டியது எதிர்ப்பிலக்கியத்தின் முக்கியமானச் செயலாக இருக்கிறது. மூன்றாவதாக சமூக அதிர்ச்சி என்ற வகையில் அல்லாமல் எதிர்ப்பு நடவடிக்கைகளைப் பொது உளவியலாக்க முயல்வது முக்கியமாகும்.மாந்தர்கள் அனைவரிடமும் உள்ளுறைந்து போயிருக்கும் எதிர்ப்பு நடவடிக்கைகள் துல்லியபடுகின்ற போது அதிகாரம் உட்பட்ட அனைத்து ஆதிக்க மனப்பான்மை மறையத் துவங்கும் .அந்த வகையில் மீறல் குறித்தப் புதிய வரைவிலக்கணத்தை முன்னெடுத்துச் செல்வது சாத்தியமாகும் நிறத்தைக் காரணம் காட்டி ஒடுக்கப்பட்ட பெண்களின் உட்குரலை கலகக் குரலை பதிவு செய்யத் தொடங்குதல் மிகவும் தேவையான ஒன்றாகும். எதிர் விளைவும், கலகமும் அழகியல் மாதிரிகளாகிறது.

\section{முடிவுரை}

தமிழக மக்களின் பிரதான உணவாகவும், எதிர் பண்பாடு என்ற அடிப்படையிலும், மாமிச உணவு குறித்த அரசியல் தமிழ்ச் சூழலில் அதிகம் விவாதிக்கபட்டுவருகிறது அதன் தொடர்ச்சியாக இதை விளக்க முடியும். மனிதனின் அடிப்படைத் தேவைகளில் உணவிற்குப் பிரதான இடம் இருந்தபோதிலும், அது குறித்த கூறுகளை விவாதிப்பதற்கு ஆர்வம் காட்டப்படுகிறது. பண்பாட்டு விவாதங்களில் அதிகக் கவனம் பெறாத ஒரு பொருளாக உணவு உள்ளது அதுவே சிலநேரங்களில் வேறுபாடுகளை உருவாக்குகிறது. தர்ம வியாதர்னு ஒரு துறவியை கௌசிக முனிவர் சந்திச்சு பல தத்துவ விளக்கங்கள் கேட்கிறார். அதே தர்ம வியாதர் காலையில் சந்தையில் மாட்டு இறைச்சி விக்கிறார். ‘அய்யா, உயிர்க்கொலை பாவம் இல்லையா?'னு கௌசிக முனிவர் கேட்டார். ‘எங்க பரம்பரை தொழிலை நான் செய்றேன். இதில் தப்பு இல்லை. ஒரு ஆட்டைக் கொலை பண்ணா, ஒரு உயிரு தான் போகுது. ஒரு கை சாதத்துல உயிருள்ள சுமார் ஐயாயிரம் நெல் வெந்து அரிசியாகியிருக்கு. ஒரு நெல்லும் உயிருள்ளது தான். ஒரு ஆடும் உயிர் உள்ளது தான். நெல்லுக்கு உயிர் இருக்குன்னு நாம சாப்பிடாம இருக்க முடியுமா?. உயிர்க்கொலை தவிர்க்க முடியாது'ன்னு சொன்னாராம். இது மகாபாரத கூற்று. "கோழி அடிச்சு கொழம்பு வெச்சு சாப்பிடு. எல்லாம் சரியாப் போகும்" என்பது வயது முதிர்ந்தவர்களின் கூற்றாக இருக்கிறது. இதில் அர்த்தம் இல்லாமல் இல்லை. உடல் பலவீனத்துக்கு கோழிக்கறி சரியான மாற்று என அறிந்துவைத்திருந்தார்கள் நம் முன்னோர். "கொன்றால் பாவம் தின்றால் போகும்" என்பது பழமொழியும் வழக்கில் உண்டு. அசைவ உணவு சாப்பிடுபவர்கள் சைவ உணவு சாப்பிடுபவர்களின் விமர்சனத்திற்கு மாற்றாக எதிரான பல்வேறு கதையாடல்களை உருவாக்கி வருகின்றனர் அசைவ உணவு சாப்பிடுபவர்கள் மீது தொடுக்கப்படும் கேள்விகளுக்கு தங்கள் பக்க எதிர் விவாதத்தை எடுத்து கூற வேண்டிய சூழலில் உள்ளனர் ஜெகதீஸ் சந்திரபோஸ் தாவரங்களுக்கும் உயிர் உண்டு என்று நிரூபித்த பின்பும் சைவம் அசைவம் என்ற வேறுபாடு தேவையறறது. சைவம் சாப்பிடுபவர்கள் உயர்ந்தவர் என்றும் அது பெருமைக்குரிய விஷயம் என்று சிந்திப்பது ஏற்றுக்கொள்ள முடியாதது. அசைவ உணவு உண்பவர்கள் தங்களுடைய எதிர்வினையாக சைவ உணவு உண்பவர்களுக்கு ஒரு பதிலை குறிப்பிடுகின்றனர். அதாவது, அசைவ உணவு உண்பவர்கள் விலங்குகளை கொல்லும் போதும் உணவுக்காக விலங்குகளை வேட்டையாடும் போதும் விலங்குகளால் தப்பி ஓடமுடியும். தனது எதிர்ப்பை சத்தமிட்டு வெளிப்படுத்த முடியும். ஆனால் தாவரங்கள் ஓடத் திராணியின்றி சத்தமும் எழுப்ப முடியாமல் ஒரு இடத்திலேயே நிற்கும். அப்போது அதன் கிளைகளை ஒடித்து உணவாக உட்கொள்ளும் உங்களை விட நாங்கள் எவ்வளவு உயர்ந்தவர்கள் என்று அசைவ உணவு உண்பவர்கள் ஒரு கதையாடலை உருவாக்கி தத்துவத்துடன் பதிலளிக்கின்றனர் இந்த விளக்கம் சமூகத்தில் ஏற்றுக்கொள்ளப்படுவதில் மாறுபட்டக் கருத்துகள் உண்டு ஆனால் சைவத்திற்கு எதிரான இவ்வகையான கதையாடல்கள் அசைவ உணவு உண்பவர்களின் நியாயத்தை உறுதி செய்யும் என்பதில் சந்தேகமில்லை. இத்தகைய வழக்காறுகளும் மற்றும் இது தொடர்பான 
கதைகளைப் போன்று ஒரு குறிப்பிட்ட குழுவின் பண்பாட்டு மரபுக்கூறுகளை எதிர்க்கும் அல்லது கேலிக்குள்ளாக்கும் எதிர்வழக்காற்று மரபைச் சார்ந்தவையாக அமையும். இ இவ்வாறே எதிர்வழக்காற்று மரபு பழமொழிகள், பாடல்கள் போன்ற பிற இலக்கிய வகைகளிலும் காணப்படுகின்றன ஒரு குறிப்பிட்ட குழு தாழ்த்தப்பட்டது என்று பழிக்கப்படும்போது அது தன்மீது ஏற்பட்ட களங்கத்தை மாற்ற தங்கள் குழு உயர்ந்தது எனச் சுட்டிக்காட்டுகிறது. இதற்கு எதிர்வழக்காற்று மரபு கருவியாகப் பயன்படுத்தப்படும்.

\section{References}

Paramasivan, T. (2009) Ariyapadatha thamizhagam, Kalachuvadu pathippagam, Chennai, India.

Stephen, G., (1999) Panpattu vergalai thedi, Nattar Forensic Research Center, Palayankottai, India.

Funding: NIL

Acknowledgement: NIL

Conflict of Interest: NIL

About the License:

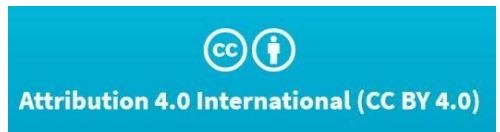

(C) The author 2021. The text of this article is licensed under a Creative Commons Attribution 4.0 International License 